\title{
Evolution of Magnetoelectric Properties of Sc-diluted $\mathrm{TbMnO}_{3}$
}

\author{
V. Cuartero ${ }^{1,2^{*}}$, J. Blasco ${ }^{1}$, J. García ${ }^{1}$, J. Stankiewicz ${ }^{1}$, G. Subías ${ }^{1}$, J. A. Rodriguez- \\ Velamazán ${ }^{1,3}$ and C. Ritter ${ }^{3}$ \\ ${ }^{1}$ Instituto de Ciencia de Materiales de Aragón, Departamento de Física de la Materia \\ Condensada,CSIC-Universidad de Zaragoza, Zaragoza, 50009, Spain \\ ${ }^{2}$ ALBA Synchrotron Light Source, Cerdanyola del Vallès, Barcelona, 08290, Spain \\ ${ }^{3}$ Institut Laue-Langevin, Boîte Postale 156, 38042 Grenoble Cèdex 9, France \\ *e-mail: vcuartero@cells.es
}

\begin{abstract}
The magnetoelectric properties of the $\mathrm{TbMn}_{1-\mathrm{x}} \mathrm{Sc}_{\mathrm{x}} \mathrm{O}_{3}$ series have been studied at low temperatures by means of heat capacity, magnetic measurements and impedance spectroscopy. $\mathrm{TbMnO}_{3}$ exhibits as expected three transitions upon lowering the temperature corresponding to the magnetic ordering of the two sublattices ( $\mathrm{Mn}$ and $\mathrm{Tb}$ ) and the ferroelectric transition. Ferroelectricity disappears with Sc dilution for $\mathrm{x}>0.1$ because the non-collinear magnetic arrangement is destroyed. The dilution of Mn with a non-magnetic ion is also detrimental to the magnetic ordering of both $\mathrm{Mn}$ and $\mathrm{Tb}$ sublattices. The system evolves to a magnetic glassy state for the intermediate compositions. $\mathrm{TbScO}_{3}$ shows Sc-deficiency and long range magnetic ordering of $\mathrm{Tb}^{3+}$ moments in the $a b$-plane brought by the direct interaction between $\mathrm{Tb}^{3+}$ ions. This ordering is different from the one found in $\mathrm{TbMnO}_{3}$ due to the lack of magnetic coupling between $\mathrm{Tb}$ - and $\mathrm{Mn}$ sublattices. A small substitution of $\mathrm{Sc}$ by $\mathrm{Mn}$ in $\mathrm{TbScO}_{3}$ destroys the $\mathrm{Tb}$ ordering giving rise to a magnetic glass behavior. This effect is ascribed to the partial polarization of $\mathrm{Tb}$ sublattice by the paramagnetic $\mathrm{Mn}$ which competes with the direct $\mathrm{Tb}-\mathrm{Tb}$ exchange.
\end{abstract}

PACS numbers: 61.05.F-, 75.10.Nr, 75.85.+t, 77.22.Ch 


\section{Introduction.}

The search for alternative mechanisms that could lead to the combination of magnetic and electric long range order in the same phase is a great challenge in solid state physics [1,2]. Multiferroic materials combine and couple long range magnetic ordering and ferroelectricity [3]. In principle, these two properties should not be compatible in conventional perovskite compounds, as the first requires half-filled shells and the second empty $d$ shells (common ferroelectric (FE) materials are transition metal (TM) oxides with empty $d$ shells). Finding of new multiferroics is mostly focused on the inversion symmetry breaking predicted [4] and observed [5] in frustrated magnets. This is the case of $\mathrm{TbMnO}_{3}$, an orthorhombic distorted perovskite, space group Pbnm, where competitive magnetic interactions lead to a non-collinear antiferromagnetic (AFM) ordering of the Mn sublattice. This, in turn, induces polar lattice distortions leading to ferroelectricity (FE) below $\mathrm{T}_{\mathrm{N} 2}=\mathrm{T}_{\mathrm{FE}} \sim 27 \mathrm{~K}[6,7]$. This magnetic behavior is rather particular and not found in many systems. In our attempt to promote FE independently of magnetic ordering, we have substituted $\mathrm{Mn}^{3+}$ with a $d^{0}$ isovalent ion such as $\mathrm{Sc}^{3+}$ in $\mathrm{TbMnO}_{3}$. In this way, we try to shed some shed light on the so-called " $d^{0}$ vs $d^{n}$ problem" [8]. Our goal is to determine whether (i) $p$ - $d$ hybridization or (ii) competitive magnetic interactions are more important to enhance a FE state.

In this context one has to mention that a ferromagnetic (FM) ground state has been found in intermediate compositions of isostructural perovskites doped with non-magnetic ions such as $\mathrm{LaMn}_{1-}$ ${ }_{x}(\mathrm{Ga} / \mathrm{Sc})_{\mathrm{x}} \mathrm{O}_{3}$ [9,10]. The origin of $\mathrm{FM}$ is not well understood so far. The proposed theoretical models, spin flipping of the Mn 3d eg-orbitals [11] and the vibronic superexchange interaction, ${ }^{12}$ put forward the importance of dynamic Jahn-Teller distortions for the occurrence of FM interactions. However, spectroscopic results on $\mathrm{LaMn}_{1-\mathrm{x}} \mathrm{Ga}_{\mathrm{x}} \mathrm{O}_{3}$ samples revealed that the onset of $\mathrm{FM}$ is concomitant with the formation of regular $\mathrm{MnO}_{6}$ octahedra [13]. Moreover, in the $\mathrm{TbMn}_{1-\mathrm{x}} \mathrm{Sc}_{\mathrm{x}} \mathrm{O}_{3}$ series the dilution with regular $\mathrm{ScO}_{6}$ octahedra does not remove the Jahn-Teller distortion of the $\mathrm{MnO}_{6}$ octahedra along the whole series and FM long-range ordered state has not been found on the intermediate composition $(\mathrm{x}=0.5)[14,15]$ In this paper we present a thorough study of the macroscopic magnetic properties of the whole series in order to completely characterize its magnetic behaviour, also including a complete determination of the magnetic ground structure of one end member of the series, $\mathrm{TbSc}_{0.94} \mathrm{O}_{3}$, with a small cation deficiency, not studied previously.

\section{Experimental Section.}

The powdered samples of $\mathrm{TbMn}_{1-\mathrm{x}} \mathrm{Sc}_{\mathrm{x}} \mathrm{O}_{3}(\mathrm{x}=0,0.05,0.1,0.2,0.3,0.4,0.5,0.6,0.7,0.8,0.9$, 1) were prepared by solid state reaction as reported elsewhere ${ }^{14}$. All samples were checked by x-ray diffraction at room temperature using a Rigaku D/max-B diffractometer with a rotating anode, selecting the $\mathrm{Cu} K_{\alpha}$ wavelength $(\lambda=1.5418 \AA$ ). All samples were single phases with exception of few batches with impurities of $\mathrm{MnO}$ or $\mathrm{Sc}_{2} \mathrm{O}_{3}$. The latter impurity always appears in $\mathrm{TbScO}_{3}$ prepared 
from stoichiometric amounts of $\mathrm{Tb}_{4} \mathrm{O}_{7}$ and $\mathrm{Sc}_{2} \mathrm{O}_{3}(\sim 2 \%$ in wg) suggesting a $\mathrm{Sc}$ deficiency for this perovskite.

We have performed heat capacity measurements using a PPMS module (Quantum Design). Small and thin pieces of pellets have been used to perform these measurements between $2 \mathrm{~K}$ and 60 K. Neutron diffraction patterns have been collected at the ILL (Grenoble). The high resolution diffractometer D2B $(\lambda=1.59 \AA)$ was used to perform the structural study of the whole series and the high flux D1B $(\lambda=2.52 \AA)$ instrument was used to determine the magnetic structure of $\mathrm{TbScO}_{3}$. Magnetic measurements have been carried out in a commercial squid magnetometer (MPMS, Quantum Design). The dc magnetization has been studied between $4 \mathrm{~K}$ and $300 \mathrm{~K}$, with hysteresis loops at $5 \mathrm{~K}$ and $-5 \mathrm{~T} \leq \mu_{0} \mathrm{H} \leq 5 \mathrm{~T}$ were also performed. In addition, we have explored the magnetic dynamical behavior by means of ac-susceptibility measurements at low temperatures, being $\mathrm{H}_{0}=4$ Oe, at frequencies ranging between $2 \mathrm{~Hz}$ and $1 \mathrm{kHz}$. All magnetic measurements were made on spherical syntherized samples.

Finally, we used an impedance analyzer to determine the dielectric constant of $x=0,0.05$ and 0.1 samples. To this end, we prepared pellets of $5 \mathrm{~mm}$ in diameter and $1 \mathrm{~mm}$ thick with silver paint electrodes. The measurements have been performed at low temperatures and $1 \mathrm{kHz}$.

\section{Results and discussion.}

All samples show a orthorhombic perovskite structure, space group Pbnm, as it has been described in a previous paper [14]. The volume of the unit cell increases as Sc concentration increases as expected from the larger size of $\mathrm{Sc}^{3+}$ with respect to $\mathrm{Mn}^{3+}$ [16]. However, the $<\mathrm{B}-\mathrm{O}-\mathrm{B}>$ bond angles $(\mathrm{B}=\mathrm{Mn}, \mathrm{Sc})$ decrease with increasing Mn-substitution. Both features, Mn-dilution and $<\mathrm{B}-\mathrm{O}-\mathrm{B}>$ decrease, are expected to be detrimental for magnetic correlations. The evolution of heat capacity with temperature is shown in Figure 1 for selected samples. Three anomalies can be appreciated for $\mathrm{TbMnO}_{3}$, as described in literature [6]. The first $\lambda$-type anomaly corresponds to the AFM sinusoidally modulated ordering of Mn moments $\left(T_{N 1} \sim 41 \mathrm{~K}\right)$. The second $\lambda$-type anomaly appears at $\mathrm{T}_{\mathrm{N} 2}=\mathrm{T}_{\mathrm{FE}} \sim 26 \mathrm{~K}$ and coincides with the transition to a non-collinear magnetic state which breaks the spatial inversion symmetry and allows ferroelectricity [5]. At $\mathrm{T}_{\mathrm{Tb}} \sim 7 \mathrm{~K}$ corresponding to the third anomaly, the Tb moments order on an incommensurate AFM ordered structure.

The substitution of $\mathrm{Mn}^{3+}$ by a nonmagnetic ion such as $\mathrm{Sc}^{3+}$ gives rise to three main variations in heat capacity measurements. First, the dilution weakens $\mathrm{Mn}^{3+}-\mathrm{O}-\mathrm{Mn}^{3+}$ interactions, as observed for other nonmagnetic ions [14,17]. This effect shows up as a strong entropy-content decrease of the first peak in Fig. 1 (a) and (b) $\left(\mathrm{T}_{\mathrm{N} 1}\right)$, which is nearly noticeable for $\mathrm{x}=0.1$ sample. In this case the transition temperature lowers down to $32 \mathrm{~K}$, in agreement with previous neutron diffraction results [14]. Second, the peak associated with the onset of FE and non-collinear magnetic ordering disappears for $\mathrm{x} \geq 0.1$. However, the presence of a low temperature peak $\left(\mathrm{T}_{\mathrm{N} 2}=\mathrm{T}_{\mathrm{FE}}\right)$ cannot 
be discarded for $\mathrm{x}=0.1$ due to the obscured by the broad peak ascribed to $\mathrm{Tb}$ correlations, so the evolution of FE transition will be checked carefully by impedance measurements.

At last, the intensity of the Tb-peak decreases strongly and broadens upon dilution of the Mn sublattice by Sc. It seems that this peak tends to a Schottky anomaly even for very low Sc concentrations $(\mathrm{x}=0.1)$. Broad anomalies in $\mathrm{C}_{\mathrm{p}} / \mathrm{T}$ curves are still seen up to $\mathrm{x}=0.4$. For high $\mathrm{x}$-values $(\mathrm{x} \geq 0.5)$, an increase of heat capacity is observed at very low temperatures $(\mathrm{T}<2.5 \mathrm{~K})$. In addition, its magnitude rises with increasing Sc content (see Fig. 1b). This may be related to the magnetic interaction of only $\mathrm{Tb}^{3+}$ moments that seems to be favoured in the $\mathrm{TbSc}_{0.94} \mathrm{O}_{3}$ lattice as suggested by neutron diffraction experiments discussed below.

Heat capacity measurements confirm the results obtained previously by neutron diffraction [14]: namely that Mn moments do not show long-range order in $x \geq 0.2$ Sc-doped samples. This is in contrast to what is observed in other orthorhombic perovskites where around $1 / 3$ of magnetic cations is enough to produce percolation and long range ordering [17, 18]. Figure 2 shows representative neutron patterns collected at at $2 \mathrm{~K}$ and $50 \mathrm{~K}$ for compositions $\mathrm{x}=0.3$ and 0.9 . The comparison of the neutron data taken at these two temperatures does not reveal the existence of long range magnetic ordering for compounds with $0.3 \leq \mathrm{x} \leq 0.9$. However, an anomalous background or diffuse scattering centered at around $2 \theta=20^{\circ}$ can be appreciated at low temperatures, for $x=0.3$ and 0.5 doped samples (see Fig. 2a for $\mathrm{x}=0.3$ ). This anomalous scattering is narrower and shifted to lower angles for $\mathrm{x}=0.7$ and 0.9 samples (see Fig. $2 b$ for $x=0.9$ ). Such a diffuse scattering has been ascribed to the short range ordering of $\mathrm{Tb}^{3+}$ moments in related compounds [17]. The shift of this scattering might arise from a change in the short-range magnetic structure of $\mathrm{Tb}$ moments. In order to gain some insight, the formal $\mathrm{TbScO}_{3}$ sample was investigated. In this compound, as seen in Fig. 3, new magnetic peaks show up at low temperatures $(\mathrm{T}=1.5 \mathrm{~K})$, which are not observed at $10 \mathrm{~K}$. The position of the first strong magnetic peak corresponds to the position of the short range order diffusion seen in the $10 \%$ doped compound (Figure 2b). The magnetic reflections correspond to A and G-type reflections, following Bertaut's notation [21]. The inset of Fig. 3 shows the Rietveld refinement of the diffraction pattern of $\mathrm{TbScO}_{3}$ collected at $1.5 \mathrm{~K}$. Refined structural parameters of two measurements, above and below the magnetic transition, are summarized in table I. Three different phases are used to get the optimum fit at $1.5 \mathrm{~K}$ : the nuclear phase (Pbnm, orthorrombic perovskite), the magnetic phase and a tiny impurity of $\mathrm{Sc}_{2} \mathrm{O}_{3}$. The fit is very good but we have found that refinement of the perovskite phase using the right stoichiometry yields unphysical negative isotropic temperature factors $\left(\mathrm{B}_{\text {iso }}\right)$ for $\mathrm{Tb}$ and $\mathrm{O}$ atoms. The refinement the Sc occupancy results in a similar fit with physical $\mathrm{B}_{\text {iso }}$ and a small cation deficiency at the $\mathrm{Sc}$ position. Our results suggest that the correct stoichiometry for this perovskite should be $\operatorname{TbSc}_{0.94(2)} \mathrm{O}_{3}$. Accordingly, the oxidation state of the $\mathrm{Tb}$ is slightly above $3+$. This agrees with the $\mathrm{Sc}_{2} \mathrm{O}_{3}$ segregation obtained in the synthesis when starting from a stoichiometric mixture.

Regarding the magnetic structure, the magnetic peaks agree with the antiferromagnetic structure $A_{x} G_{y}$, corresponding to the $\Gamma_{2}$ irreducible representation for the Tb atom located at the $4 \mathrm{c}$ 
Wyckoff position with $\mathbf{k}=(0,0,0)$. A similar ordering has been reported for $\mathrm{TbCrO}_{3}$ and $\mathrm{TbCoO}_{3}$ [22]. The magnetic moments order in the $a b$-plane in agreement with the strong planar anisotropy observed in Tb-based perovskites. The refined magnetic components are $\mu_{\mathrm{x}}=5.22$ and $\mu_{\mathrm{y}}=5.71$. Therefore, the moments are located close to the diagonals in the $a b$-plane $\left(47.6^{\circ}\right.$ respect to the $\mathrm{x}$ axis). The total magnetic moment obtained for $\mathrm{Tb}$ is 7.74(4) $\mu_{\mathrm{B}}$, which is in agreement with the values observed in other related Tb-perovskite oxides [22].

According to the neutron diffraction results, already $10 \%$ of $\mathrm{Mn}$ on the $\mathrm{Sc}$ sublattice $\left(\mathrm{TbMn}_{0.1} \mathrm{Sc}_{0.9} \mathrm{O}_{3}\right)$ suppresses the $\mathrm{Tb}$ long range ordering observed in $\mathrm{TbScO}_{3}$. This suggests that the direct exchange $\left(\mathrm{J}_{\mathrm{Tb}-\mathrm{Tb}}\right)$ is weaker than the interaction arising from the local field induced by the paramagnetic impurity $\left(\mathrm{J}_{\mathrm{Mn}-\mathrm{Tb}}\right)$. Similar results have been found in related perovskites like $\mathrm{NdFe}_{1}$ ${ }_{\mathrm{x}} \mathrm{Ga}_{\mathrm{x}} \mathrm{O}_{3}$ or $\mathrm{TbFe}_{1-\mathrm{x}} \mathrm{Al}_{\mathrm{x}} \mathrm{O}_{3}$ where the presence of magnetic impurities in the non-magnetic sublattice $(\mathrm{x} \sim 0.9)$ inhibits the long range magnetic ordering of the rare earth sublattice $[18,23]$. Our results suggest that similar effects exist in the $\mathrm{TbMn}_{1-\mathrm{x}} \mathrm{Sc}_{\mathrm{x}} \mathrm{O}_{3}$ : a small concentration of $\mathrm{Mn}^{3+}$ ions polarizes a significant amount of $\mathrm{Tb}^{3+}$ cations and inhibits the long range $\mathrm{A}_{\mathrm{x}} \mathrm{G}_{\mathrm{y}}$ ordering.

Now we turn to the results of magnetization measurements carried out on the compounds studied. The low temperature $(4 \mathrm{~K}<\mathrm{T}<80 \mathrm{~K})$ dependence of the dc magnetization under $5 \mathrm{kOe}$ magnetic field in zero field cooled (ZFC) conditions is displayed in figure 4 (a). All samples show a paramagnetic behavior and some of them also exhibit anomalies at low temperature. $\mathrm{TbMn}_{0.9} \mathrm{Sc}_{0.1} \mathrm{O}_{3}$ sample shows a broad maximum close to $8 \mathrm{~K}$, similar to the one observed in $\mathrm{TbMnO}_{3}$ [6]. This anomaly is likely related to the Tb-sublattice ordering as it does not show any variation with frequency (not shown here). As Mn is diluted with Sc, a magnetization increase is observed at low temperature. In the case of $x=0.3$, the intensity of the broad peak rises and the maximum shifts to higher temperatures. This sample shows magnetic irreversibility between ZFC and FC conditions (not shown here) and the dynamic behaviour reported later on in ac measurements suggests the existence of a magnetic glassy behaviour. $\mathrm{TbMn}_{1 / 2} \mathrm{Sc}_{1 / 2} \mathrm{O}_{3}$ shows the highest magnetization in the series at low temperature with the highest temperature for the inflection point in the magnetization curve. There is not peak at this magnetic field but it was observed using smaller strength of the field [14]. These features reveal the enhancement of FM correlations for this composition, as reported for $\mathrm{LaMn}_{0.5} \mathrm{Sc}_{0.5} \mathrm{O}_{3}$ [10], but long range $\mathrm{FM}$ ordering was discarded from neutron diffraction measurements [14]. Further dilutions $(x=0.7)$ leads to a decreases of low temperature magnetization and a sharper peak is observed at lower temperatures $(6 \mathrm{~K})$ whose occurrence seems to be also related to glassy properties. The most diluted sample $(\mathrm{x}=0.9)$ shows a paramagnetic-like behavior down to $4 \mathrm{~K}$. The strong magnetization enhancement in the lower measurement limit suggests the existence of a peak below $4 \mathrm{~K}$ as confirmed by ac measurements shown later. Such measurements will be used to characterize the dynamic behaviour observed in $0.3 \leq \mathrm{x} \leq 0.9$ compounds.

At high temperatures $(100 \mathrm{~K}<\mathrm{T}<300 \mathrm{~K})$, all samples behave as conventional paramagnets and follow a Curie-Weiss law, as shown on the inset of fig. 4 (a) for $\mathrm{x}=0.1,0.5$ and 0.9 . The parameters obtained from the fits to this law in the temperature range $90 \mathrm{~K} \leq \mathrm{T} \leq 300 \mathrm{~K}$ for all samples are 
summarized in table II. The Curie constant (C) decreases linearly with decreasing the magnetic ions concentration in the TM sublattice. The same trend is observed for effective paramagnetic moments obtained from the fit. The obtained values are in very good agreement with the calculated ones for the paramagnetic moments $\left(\rho_{\text {theo }}\right.$ ), which are calculated taking into account to the experimental values of the paramagnetic ions, that is $\rho\left(\mathrm{Mn}^{3+}\right)=4.9 \mu_{\mathrm{B}}$ and $\rho\left(\mathrm{Tb}^{3+}\right)=9.5 \mu_{\mathrm{B}}$ [24]. The total value is calculated from $\rho_{\text {theo }}^{2}=(1-\mathrm{x}) \rho^{2}\left(\mathrm{Mn}^{3+}\right)+\rho^{2}\left(\mathrm{~Tb}^{3+}\right)$. The Weiss constant $(\theta)$ is always negative, indicating importance of the AFM correlations in the paramagnetic phase. It tends to decrease as the Sc content increases, which indicates that the correlations weaken upon the dilution with a nonmagnetic ion.

Hysteresis loops measured at $5 \mathrm{~K}$ are shown in fig. 4 (b). $\mathrm{TbMn}_{0.9} \mathrm{Sc}_{0.1} \mathrm{O}_{3}$ shows a loop similar to the parent $\mathrm{TbMnO}_{3}$ with a linear increase of $\mathrm{M}$ at low $\mathrm{H}$ and a slope change at $30 \mathrm{kOe}$ resembling the metamagnetic transition of the undoped compound [17].As Mn sublattice is diluted, the magnetization rises at low field adopting the shape of a FM loop without reaching magnetic saturation at $50 \mathrm{kOe}$. The expected magnetization for a full saturated Tb sublattice is $9 \mu_{\mathrm{B}} / \mathrm{fu}$ [25] while the magnetization of $\mathrm{TbMn}_{1-\mathrm{x}} \mathrm{Sc}_{\mathrm{x}} \mathrm{O}_{3}$ samples ranges between 3.5 and $3 \mu_{\mathrm{B}} / \mathrm{fu}$ (fu stands for formula unit) at $50 \mathrm{kOe}$. The evolution of the loops along the series indicates than the main contribution to the magnetization comes from the $\mathrm{Tb}$ sublattice which is easily polarized as $\mathrm{Mn}$ content decreases because the $\mathrm{Mn}-\mathrm{Tb}$ interaction is weakened and hence, the $\mathrm{Tb}-\mathrm{Tb}$ interaction strengthened by the magnetic field prevails. It is worth remembering that magnetic field favors anisotropic ordering (with FM component) of $\mathrm{Tb}^{3+}$ moments in the ab-plane in related perovskites [17].

The low-field range is shown in the inset of figure 4 (b) for $x=0.3,0.5$ and 0.7 . It can be appreciated that there is a net remanent magnetization. The highest contribution is found for $\mathrm{x}=0.5$, where $\mathrm{M}_{\mathrm{rem}}=0.21 \mu_{\mathrm{B}} / \mathrm{fu}$ and the coercitive field is $1.2 \mathrm{kOe}$, while for $\mathrm{x}=0.3$ and 0.7 the remanence is 0.11 and $0.09 \mu_{\mathrm{B}}$ respectively, and a coercitive field of $\sim 0.5 \mathrm{kOe}$ is obtained for both samples. These samples show relatively high coercitivity, very similar to the one observed on $\mathrm{LaMn}_{1 / 2} \mathrm{Sc}_{1 / 2} \mathrm{O}_{3}$ which is a ferromagnet [15]. The large coercivity puts forward a high magnetic anisotropy in these compounds which may be related with the high disorder in the Mn-Sc sublattice.

In order to study magnetic dynamic behaviour of the samples as a function of temperature, we have performed ac magnetic susceptibility measurements at various frequencies (figures 5 and 6). For the low doping $(x \leq 0.2)$, the ac susceptibility does not depend on frequency (not shown here). However, for higher concentrations $(x \geq 0.3)$, the samples show strong frequency-dependent peaks at low temperature, as can be seen on figure 5 for $\mathrm{x}=0.3,0.4,0.6$ and 0.7 . The measurements corresponding to $\mathrm{x}=0.5$ have been reported in a previous paper [15] and also show a dynamic dependence, but its peak is quite flattened. This different behaviour may be ascribed to bigger magnetic cluster size. It is thought that $\mathrm{FM}$ interactions are optimized for $\mathrm{x}=0.5$ composition but long range magnetic ordering is not achieved here due to competition between nearest-neighbours 
and next-nearest-neighbours interactions [17]. As we will show below, the glassy magnetic behaviour of this sample is further from a canonical spin glass.

It is possible to appreciate a cusp peak on the real part of susceptibility ( $\chi^{\prime}$ ) for the rest of intermediate compositions $(0.3 \leq \mathrm{x} \leq 0.7)$. This peak depends on frequency: it shifts to higher temperatures and is less intense as the frequency increases. The out of phase component $(\chi$ ') also shifts to higher temperatures as frequency increases, while the intensity of the peaks increases for $\mathrm{x}=0.3$ and 0.4 compositions but there is not a clear trend for the other two samples ( $\mathrm{x}=0.6$ and 0.7 ). These features are the hallmarks of a glassy magnetic system [23] as we discuss in detail later on.

Regarding the Sc-rich samples, the evolution of the ac magnetic susceptibility with frequency dependence can be seen on fig. 6 ( $\mathrm{x}=0.8,0.9$ and 1). $\mathrm{TbSc}_{0.94} \mathrm{O}_{3}$,fig. 6 (c), exhibits a peak in the $\chi$ ' at $3 \mathrm{~K}$ without dynamical behaviour. This can be associated with the onset of $\mathrm{Tb}$ long-range magnetic ordering in accordance with neutron diffraction results (fig. 3). In the other two samples, a strong dynamic behavior is observed in $\chi$ ' with a cusp shifted to lower temperatures as Sc content increases. Similar shift is also observed in the imaginary component. In order to quantify the dynamic magnetic behavior of the samples, we will focus first on the peak temperature and frequency dependence, $T_{p}(v=\omega / 2 \pi)$, of $\chi^{\prime \prime}$ through the defined quantity $p=\Delta T_{p} / T_{p} \cdot \Delta(\log \omega)$, which is commonly used to classify glassy magnetic systems [26]. In the case of a metallic spin glass such as PdMn (RKKY interaction), $\mathrm{p}=0.013$, while in semiconducting spin glasses like (EuSr)S and (FeMg) $\mathrm{Cl}_{2}$ (exchange interaction) $\mathrm{p} \sim 0.06-0.1$. For $\mathrm{TbMn}_{1-\mathrm{x}} \mathrm{Sc}_{\mathrm{x}} \mathrm{O}_{3}, 0.3 \leq \mathrm{x} \leq 0.8$, the values of $\mathrm{p}$ range between $0.03 \leq p \leq 0.1$, as it can be seen on the inset of fig. 7. The value of $p$ is almost constant for Sc concentrations up to $\mathrm{x}=0.5$ ( $\mathrm{p} \sim 0.03$ ) and then it rises with the $\mathrm{Sc}$ content up to a value of $\mathrm{p} \sim 0.1$ for $\mathrm{x}=0.8$. The inflection point at $\mathrm{x}=0.5$ may be related to weaker interaction among magnetic moments as they become more distant.

The $T_{p}$ frequency shift observed in ac susceptibility (out of phase component) can be evaluated by several empirical laws depending on the nature of the freezing process. Firstly, this was fitted by a simple Arrhenius law, which is obeyed by weakly interacting or non-interacting superparamagnetic clusters and is described as follows: $\tau=\tau_{0} \exp \left[\mathrm{E}_{\mathrm{a}} / k_{\mathrm{B}} \mathrm{T}_{\mathrm{p}}\right]$. Here $\mathrm{E}_{\mathrm{a}}$ corresponds to the anisotropic energy barrier and $\tau_{0}$ is the characteristic relaxation time which describes the time scale of the dynamic fluctuations of the system. The fits to this law are not satisfactory and give unphysical values for $\tau_{0}$ and $\mathrm{E}_{\mathrm{a}}$.

On the other hand, glassy interacting magnetic systems show characteristic relaxation times which can be fitted to Vogel-Fulcher law, that is $\tau=\tau_{0} \exp \left[\mathrm{E}_{\mathrm{a}} / k_{\mathrm{B}}\left(\mathrm{T}_{\mathrm{p}}-\mathrm{T}_{0}\right)\right]$ [27]. Typically $\tau_{0} \sim 10^{-13} \mathrm{~s}$ for canonical spin glass systems, but this order of magnitude is also found in other manganites such as $\mathrm{Eu}_{1 / 2} \mathrm{Ba}_{1 / 2} \mathrm{MnO}_{3}$ where a spin glass state is observed [28]. $\mathrm{T}_{0}$ represents the clusters or spins interaction temperature. It is calculated from the linear relation between $\left(\Delta \mathrm{T}_{\mathrm{p}}(v) / \Delta \ln v\right)^{1 / 2}$ and the average temperature $<\mathrm{T}_{\mathrm{p}}>=\mathrm{T}_{\mathrm{p}}(v)+\Delta \mathrm{T}_{\mathrm{p}}(\mathrm{v}) / 2$ [29]. The values obtained for $\mathrm{T}_{0}$ are shown in table III, together with the results obtained from the Vogel-Fulcher fits, shown in figure 7 for $x=0.3,0.5,0.7$ 
and 0.8. The values of $\mathrm{T}_{0}$ are alike similar for the intermediate compounds $\mathrm{x}=0.4$ and 0.5 but it starts decreasing linearly as the Sc concentration increases further. The relaxation time accounting for the dynamical processes has approximately the same order of magnitude $\left(10^{-10}-10^{-11} \mathrm{~s}\right)$ in all compounds, but it is remarkably higher for the intermediate compound $\mathrm{TbMn}_{1 / 2} \mathrm{Sc}_{1 / 2} \mathrm{O}_{3}\left(\sim 10^{-8} \mathrm{~s}\right)$. The activation energy is also lower in this compound. Regarding the samples left ( $\mathrm{x}=0.3,0.4,0.6$ and 0.7$)$, the value of $\tau_{0}$ is larger than the relaxation time expected for an atomic scale spin-glass state, and it may be ascribed to nanosized interacting entities [30]. In the case of the intermediate compound $(x=0.5)$ the longer relaxation time is found, being $\tau_{0} \sim 10^{-8} \mathrm{~s}$. This order of magnitude for dynamical processes is expected for larger interacting entities than in the other compositions [29].

Dielectric properties have been measured in the lower diluted samples, that is, $x=0,0.05,0.1$ and 0.3 . The results for the dielectric constant at low temperatures at $1 \mathrm{kHz}$ are plotted in figure 8 . The peak observed at $\sim 25 \mathrm{~K}$ in fig. 8 (a) for $\mathrm{TbMnO}_{3}$ coincides with the ferroelectric transition previously reported [6]. When this material is diluted with a $5 \%$ of Sc, this peak is smoothed and shifts to lower temperatures $(12 \mathrm{~K})$, as it can be seen in figure 8 (b). In the case of $10 \%$ doped sample, the peak is damped, although still noticeable at $10 \mathrm{~K}$. However, no changes are observed in the dielectric constant of the $30 \%$ doped sample at low temperatures -fig. 8 (c). From these results we can assert that the ferroelectric transition rapidly disappears with non-magnetic doping on $\mathrm{TbMnO}_{3}$. This agrees with the magnetic origin of $\mathrm{FE}$ in $\mathrm{TbMnO}_{3}$ : instead of favouring FE with $d^{0}$ ions, Sc doping rapidly destroys $\mathrm{Mn}$ cycloidal ordering. Then, $\mathrm{FE}$ is forbidden by symmetry in this system. This is contrary to the behaviour observed in $\mathrm{REMn}_{1-\mathrm{x}} \mathrm{Ga}_{\mathrm{x}} \mathrm{O}_{3}$ hexagonal compounds where non-magnetic doping favoured a FE state at higher Curie temperatures. In this case the displacement of the RE ions along $c$ axis is responsible for the appearance of FE [31].

\section{Conclusions.}

We have obtained the magnetic phase diagram for the $\mathrm{TbMn}_{1-\mathrm{x}} \mathrm{Sc}_{\mathrm{x}} \mathrm{O}_{3}$ series compounds. We start at both edges of the series as it is summarized in figure 9. The Mn-rich region $(x \leq 0.1)$ shows an AFM ordering on Mn sublattice which is non-commensurate. This long-range magnetic ordering of $\mathrm{Mn}$ disappears around $\mathrm{x} \geq 0.2$. The ordering of $\mathrm{Tb}$ is also affected by the dilution of $\mathrm{Mn}$ sublattice. $\mathrm{Tb}$ magnetic ordering correlated to $\mathrm{Mn}$ ordering observed in $\mathrm{TbMnO}_{3}$, rapidly disappears and short range ordering is signed by heat capacity and neutron diffraction measurements on Sc diluted samples. In the Sc-rich region, formal $\mathrm{TbScO}_{3}$ exhibits Sc-deficiency and a long range ordering of $\mathrm{Tb}$ sublattice coming from direct $\mathrm{Tb}$ - $\mathrm{Tb}$ interaction. The Rietveld refinements are in accordance with $\mathrm{A}_{\mathrm{x}} \mathrm{G}_{\mathrm{y}}$ commensurate ordering. Small substitution of $\mathrm{Sc}$ with paramagnetic $\mathrm{Mn}^{3+}$ cations is very unfavorable for this ordering and magnetic peaks vanish in the neutron patterns of $\mathrm{TbMn}_{0.1} \mathrm{Sc}_{0.9} \mathrm{O}_{3}$ likely due to the competition between $\mathrm{J}_{\mathrm{Tb}-\mathrm{Tb}}$ and $\mathrm{J}_{\mathrm{Mn}-\mathrm{Tb}}$ interactions.

The presence of competitive magnetic interactions and structural disorder observed for intermediate concentrations gives rise to a glassy behaviour. This is tested by ac susceptibility measurements, which show peaks at different temperatures depending on the frequency of the magnetic field applied. The $T_{p}$ frequency dependence of $\chi^{\prime \prime}$ is fitted to a Vögel-Fulcher law for most 
compositions $(0.3 \leq \mathrm{x} \leq 0.8)$, giving similar results $\left(\tau \sim 10^{-10}-10^{-11} \mathrm{~s}\right)$ that point out to a non-canonical spin glass-like system with nanosized interacting entities, that is, a cluster glass (CG). The most noticeable difference is found for the intermediate compound $(\mathrm{x}=0.5)$, where a longer relaxation time $\left(\sim 10^{-8} \mathrm{~s}\right)$ and lower activation energy is found. This suggests that for this composition larger CG entities are interacting.

The disappearance of FE is intimately correlated to the lack of non-collinear ordering on $\mathrm{Mn}$ sublattice. This result confirms that competitive magnetic interactions $J_{\mathrm{Mn}-\mathrm{Mn}}$ and $\mathbf{J}_{\mathrm{Tb}-\mathrm{Mn}}$ prevail over $d$ and $p$ orbitals hybridization, even though the formation of dipoles could have been enhanced on systems diluted with $d^{0}$ - ions.

Acknowledgements. The authors thank to Servicio de difracción de rayos $\mathrm{X}$ from Zaragoza University for $x$-ray diffraction measurements. We also thank ILL and SPINS for neutron beam time allocation. Financial support from the Spanish MEC (project MAT2012-38213-C02-01) and DGA (CAMRADS) is acknowledged. 


\section{References.}

[1] Cheong S-W and Mostovoy M 2007 Nature Mat. 613

[2] Ramesh R and Spalding N A 2007 Nature Mat. 621

[3] Schmid H 1994 Ferroelectrics 162665

[4] Sergienko I A and Dagotto E 2006 Phys. Rev. B 73094434

[5] Kenzelmann M, Harris A B, Jonas S, Broholm C, Schefer J, Kim S B, Zhang C L, Cheong S-W, Vajk OP and Lynn J W 2005 Phys. Rev. Lett. 95087206

[6] Kimura T, Goto T, Shintani H, Ishizka K, Arima T and Tokura Y 2003 Nature (London) 42655

[7] Walker H C, Fabrizi F, Paolasini L, de Bergevin F, Herrero-Martín J, Boothroyd A T, Prabhakaran D, McMorrow D F 2011 Science 3331273

[8] Khomskii D 2009 Physics 220

[9] Blasco J, García J, Campo J, Sánchez M C and Subías G 2002 Phys. Rev. B 66174431

[10] Goodenough J B, Dass R I and Zhou J 2002 Solid State Sci.4 297

[11] Farrell J and Ghering G A 2004 New J. Phys 6168

[12]Zhou J-S and Goodenough J B 2003 Phys. Rev. B 68144406

[13]Sánchez M C, Subías G, García J and. Blasco J 2004 Phys. Rev. B 69, 184415

[14]Cuartero V, Blasco J, García J, Subías G, Ritter C and Rodríguez-Velamazán J A 2010 Phys. Rev. B $\mathbf{8 1} 224117$

[15]Blasco J, Cuartero V, García J and Rodríguez-Velamazán J A 2012 J. Phys: Cond. Matt. 24

076006

[16]Shannon R D Acta Cryst. 1976 A32 751

[17]Cuartero V, Blasco J, Rodríguez-Velamazán J A, García J, Subías G, Ritter C, Stankiewicz J and Canadillas-Delgado L 2012 Phys. Rev. B 86104413

[18]Parra-Boderías M, Bartolomé F, Rodríguez Velamazán J A and Bartolomé J 2011 J. Phys: Cond. Matt. 23046003

[19]Malo S, Maignan A, Hervieu M, Poeppelmeier K R and Raveau B 2005 Solid State Sci. 71492. [20]Prokhnenko O, Aliouane N, Feyerherm R, Dudzik E, Wolter A U B, Maljuk A, Kiefer K, and Argyriou D N 2010 Phys. Rev. B 81024419

[21]Bertaut E F, Acta Cryst. 1968 A24 217

[22]Mareschal J, Sivardière J and De Vries G F 1968 J. of Appl. Phys. 3921364

[23]Bombik A, Lesniewska B, Mayer J, Oles A, Pacyna A W, Przewoznik J, 1997 J. Magn. Magn. Mat. 168139

[24]Kittel C. Introduction to Solid State Physics. 2005 7Th Edition

[25]Quezel S, Techeou F, Rossat-Mignod J, Quezel G and Roudaut E 1977 Physica B \& C 86-88 916

[26]Mydosh J A Spin glasses. An experimental introduction. 1993 Taylor and Francis

[27]Tholence J L, Solid State Commun. 199388917

[28]Mathieu R and Tokura Y 2007, J. Phys. Soc. Jap. 76, 124706. Mathieu R, Akahoshi D, Asamitsu A, Tomioka Y and Tokura Y 2004 Phys. Rev. Lett. 93, 227202

[29]Souletie J and Tholence J L 1992, Phys. Rev. B 32, 516

[30]Mukadam M D, Yusuf S M, Sharma P, Kulshreshtha S K and Dey G K 2005 Phys. Rev. B 72 174408

[31]Zhou H D, Denyszyn J C, and Goodenough J B 2005 Phys. Rev. B 72224401 


\section{TABLES}

Table I. Refined lattice parameters, fractional atomic coordinates, isotropic temperature factors, magnetic moment and reliability factors for $\mathrm{TbSc}_{0.94} \mathrm{O}_{3}$ at 10 and $1.5 \mathrm{~K}$.

\begin{tabular}{r|c|c}
\hline $\mathrm{T}(\mathrm{K})$ & 1.5 & 10 \\
\hline $\mathrm{a}(\AA)$ & $5.5063(1)$ & $5.5057(1)$ \\
$\mathrm{b}(\AA)$ & $5.7607(1)$ & $5.7605(1)$ \\
$\mathrm{c}(\AA)$ & $7.9648(2)$ & $7.9629(2)$ \\
$\mathrm{Vol}\left(\AA^{3}\right)$ & $252.65(1)$ & $252.54(1)$ \\
$\mathrm{Tb}:(\mathrm{x} \mathrm{y} 1 / 4)$ & & \\
$\mathrm{x}$ & $-0.0173(4)$ & $-0.0174(4)$ \\
$\mathrm{y}$ & $0.0595(2)$ & $0.0592(3)$ \\
$\mathrm{B}_{\text {iso }}\left(\AA^{2}\right)$ & $0.08(6)$ & $0.05(5)$ \\
$\mu \mathrm{x}\left(\mu_{\mathrm{B}}\right)$ & $5.22(2)$ & - \\
$\mu \mathrm{y}\left(\mu_{\mathrm{B}}\right)$ & $5.71(2)$ & - \\
$\mathrm{Sc}:\left(01 \frac{1}{2} 0\right)$ & & \\
$\mathrm{B}_{\text {iso }}\left(\AA^{2}\right)$ & $0.16(6)$ & $0.24(4)$ \\
$\mathrm{O} 1:(\mathrm{x} \mathrm{y} 1 / 4)$ & & \\
$\mathrm{x}$ & $0.1241(6)$ & $0.1243(5)$ \\
$\mathrm{y}$ & $0.4452(7)$ & $0.4446(5)$ \\
$\mathrm{B}_{\text {iso }}\left(\AA^{2}\right)$ & $0.39(11)$ & $0.38(9)$ \\
$\mathrm{O} 2:(\mathrm{x} \mathrm{y} \mathrm{z)}$ & & \\
$\mathrm{x}$ & $0.6912(5)$ & $0.6917(3)$ \\
$\mathrm{y}$ & $0.3062(5)$ & $0.3064(3)$ \\
$\mathrm{z}$ & $0.06949(3)$ & $0.6467(2)$ \\
$\mathrm{B}_{\text {iso }}\left(\AA^{2}\right)$ & $0.60(10)$ & $0.73(8)$ \\
$\mathrm{R}_{\mathrm{wp}}(\%)$ & 2.1 & 1.5 \\
$\mathrm{R}_{\mathrm{Bragg}}(\%)$ & 0.7 & 0.8 \\
$\mathrm{R}_{\text {mag }}(\%)$ & 0.9 & - \\
$\chi^{2}$ & 3.8 & 1.9 \\
\hline
\end{tabular}

Table II. Magnetic parameters - Curie constant, Weiss parameter, experimental and calculated effective paramagnetic moments- obtained from fits to a Curie-Weiss law.

\begin{tabular}{|l|c|c|c|c|}
\hline $\mathrm{x}$ & $\mathrm{C}(\mathrm{emu} \cdot \mathrm{K} / \mathrm{mol})$ & $\theta(\mathrm{K})$ & $\rho_{\mathrm{ef}}\left(\mu_{\mathrm{B}} / \mathrm{f} . \mathrm{u}.\right)$ & $\rho_{\text {theo }}\left(\mu_{\mathrm{B}} /\right.$ f.u. $)$ \\
\hline 0.1 & $14.07(2)$ & $-28.9(5)$ & $10.61(1)$ & 10.58 \\
\hline 0.3 & $13.93(2)$ & $-21.8(6)$ & $10.57(1)$ & 10.35 \\
\hline 0.5 & $13.35(5)$ & $-14.6(8)$ & $10.34(3)$ & 10.11 \\
\hline 0.7 & $12.70(3)$ & $-7.8(6)$ & $10.08(2)$ & 9.87 \\
\hline 0.9 & $11.04(2)$ & $-9.8(5)$ & $9.40(1)$ & 9.63 \\
\hline 1 & $10.27(3)$ & $-12.6(2)$ & $9.06(2)$ & 9.5 \\
\hline
\end{tabular}


Table III. Magnetic dynamic parameters obtained from the fitting of ac susceptibility data to a Vogel-Fulcher law. $\mathrm{T}_{0}$ corresponds to the clusters interacting temperature, $\tau_{0}$ is the relaxation time of the dynamical processes and $\mathrm{E}_{\mathrm{a}}$ is the activation energy barrier the clusters have to overcome to be aligned with the field.

\begin{tabular}{|c|c|c|c|}
\hline $\mathrm{x}$ & $\mathrm{T}_{0}(\mathrm{~K})$ & $\tau_{0}(\mathrm{~s})$ & $\mathrm{E}_{\mathrm{a}} / \mathrm{k}_{\mathrm{B}}(\mathrm{K})$ \\
\hline 0.3 & 12.1 & $2.8 \cdot 10^{-11}$ & 91 \\
\hline 0.4 & 10.7 & $2.0 \cdot 10^{-10}$ & 90 \\
\hline 0.5 & 10.1 & $1.1 \cdot 10^{-8}$ & 45 \\
\hline 0.6 & 7.27 & $3.6 \cdot 10^{-10}$ & 106 \\
\hline 0.7 & 4.36 & $8.2 \cdot 10^{-11}$ & 127 \\
\hline 0.8 & 2.03 & $4.3 \cdot 10^{-10}$ & 101 \\
\hline
\end{tabular}




\section{FIGURES}

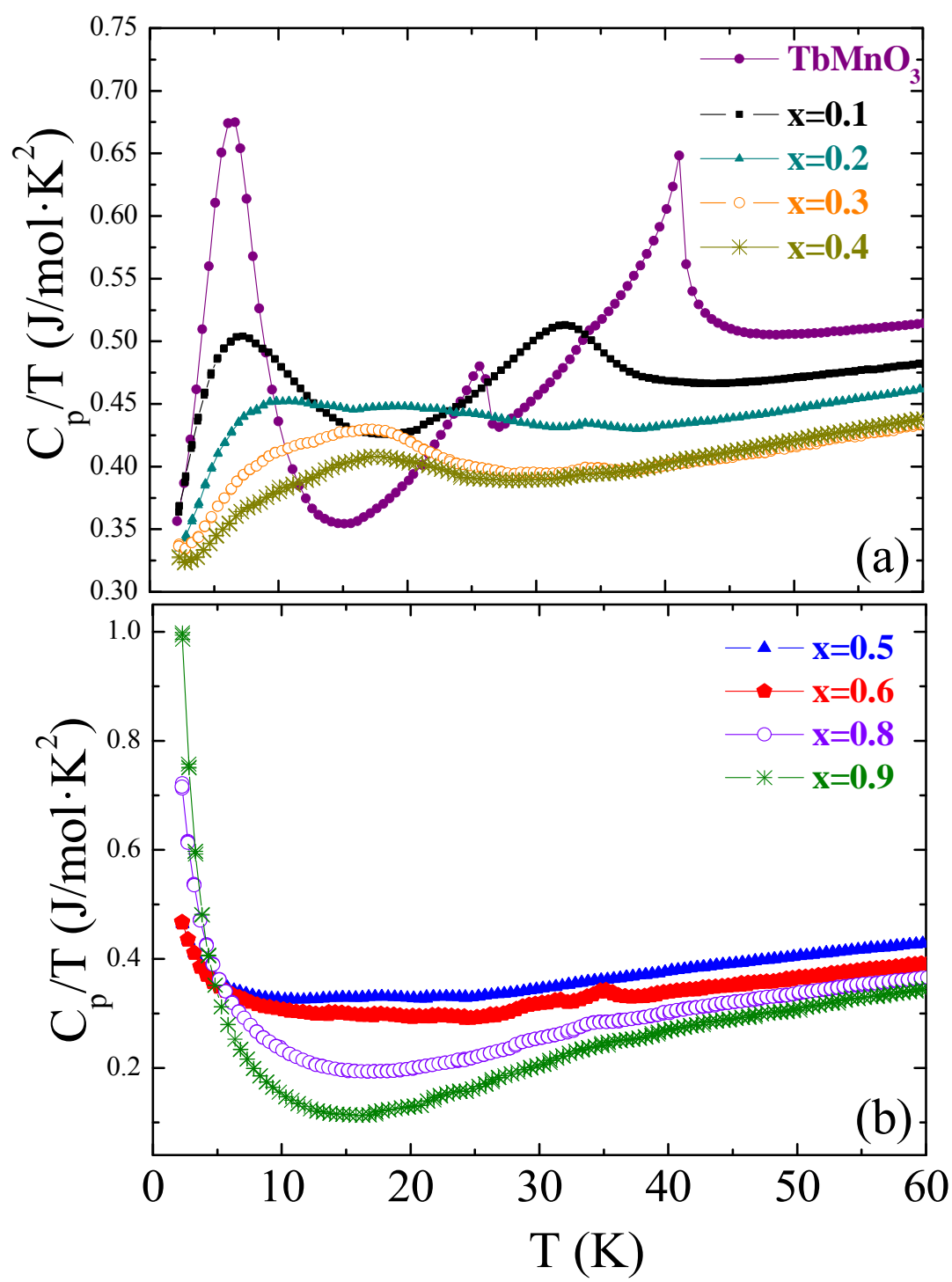

Fig. 1. Specific heat divided by temperature for (a) low diluted $(x \leq 0.4)$ and (b) high diluted $(x \geq 0.5)$ samples of $\mathrm{TbMn}_{1-\mathrm{x}} \mathrm{Sc}_{\mathrm{x}} \mathrm{O}_{3}$ series. 

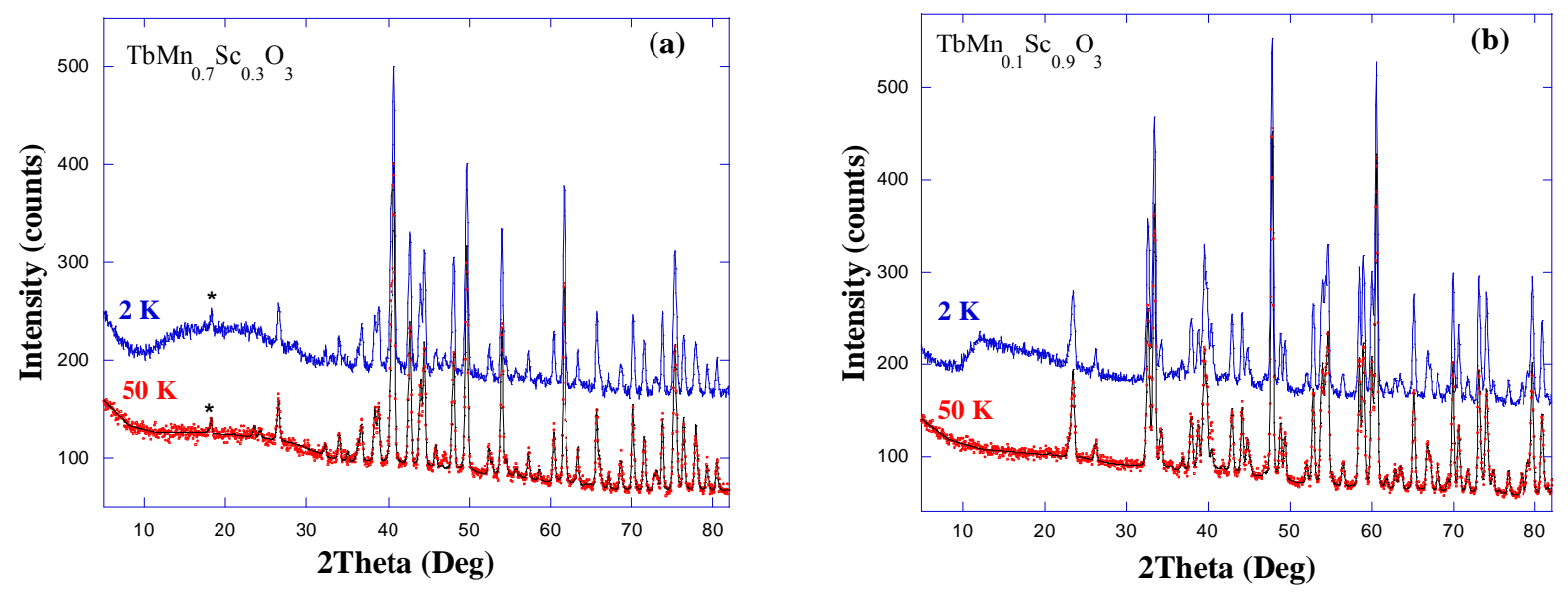

Fig. 2. Details of the neutron diffraction patterns measured at $\mathrm{D} 2 \mathrm{~B}(\lambda=1.59 \AA)$ for (a) $\mathrm{TbMn}_{0.7} \mathrm{Sc}_{0.3} \mathrm{O}_{3}$ and (b) $\mathrm{TbMn}_{0.1} \mathrm{Sc}_{0.9} \mathrm{O}_{3}$ samples at 50 and $2 \mathrm{~K}$ (shifted on $\mathrm{y}$-axis). The points and line in the measurements at $50 \mathrm{~K}$ stand for experimental and calculated pattern respectively. (a) The asterisk marks the main magnetic peak from a $\mathrm{MnO}$ impurity $(\sim 1 \%$ in weight $)$ with $\mathrm{T}_{\mathrm{N}} \sim 110 \mathrm{~K}$. 


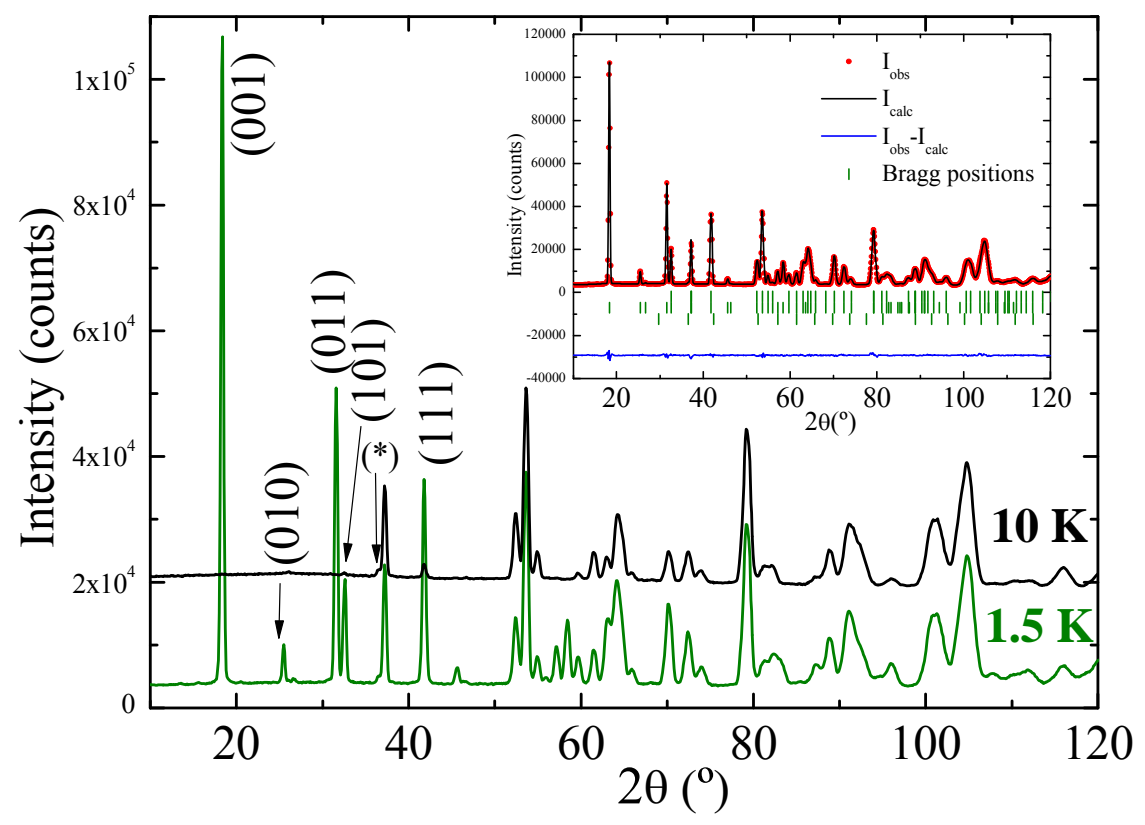

Fig. 3. Neutron diffraction patterns collected at D1B $(\lambda=2.52 \AA)$ above and below the transition temperature of $\mathrm{Tb}$ sublattice in formal $\mathrm{TbScO}_{3}$. The asterisk refers to the peak corresponding to the impurity of $\mathrm{Sc}_{2} \mathrm{O}_{3}(2 \%$ in wg). Inset: Rietveld refinement of the pattern at $1.5 \mathrm{~K}$.
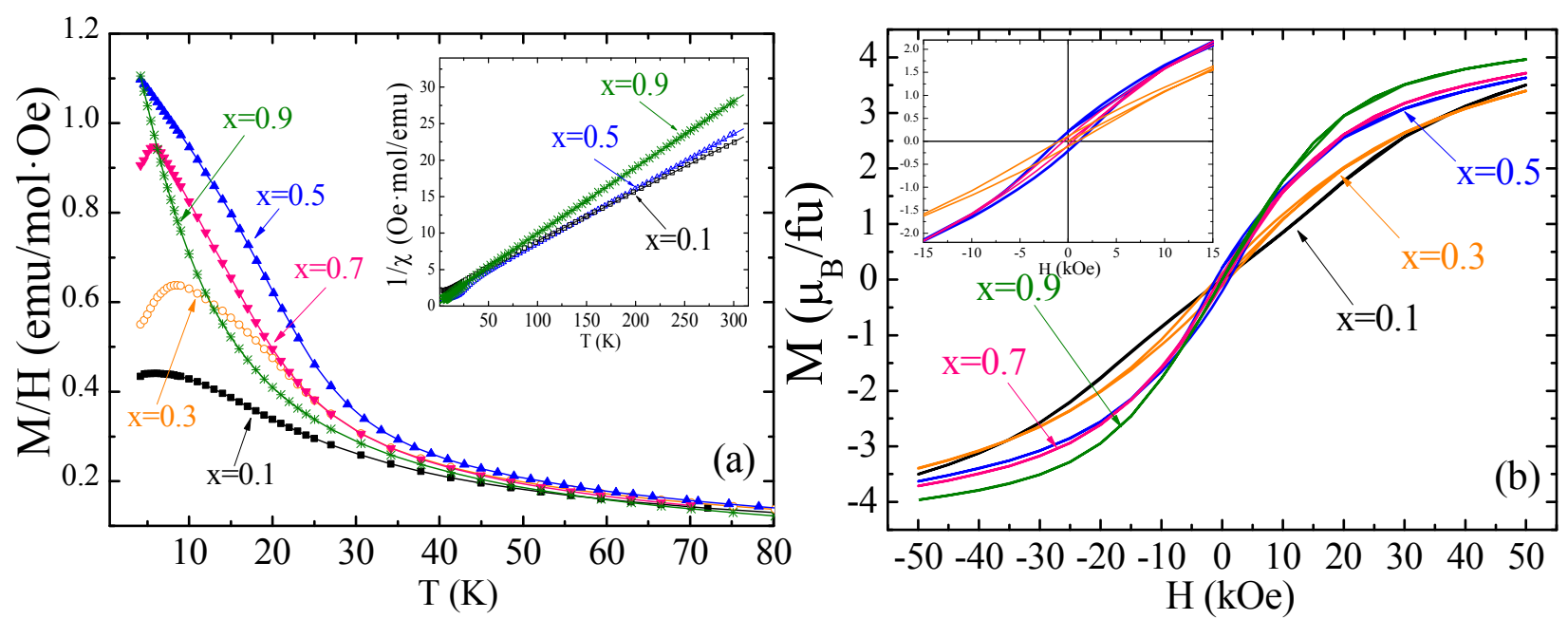

Fig. 4 (a) dc magnetization temperature dependence for $\mathrm{TbMn}_{1-\mathrm{x}} \mathrm{Sc}_{\mathrm{x}} \mathrm{O}_{3}(\mathrm{x}=0.1,0.3,0.5,0.7$ and 0.9) samples, applying 0.5 T magnetic field. Inset: Curie-Weiss law fit at high temperatures for selected samples. (b) Hysteresis loops from the same samples, at $\mathrm{T}=5 \mathrm{~K}$. Inset: zoom of the region $1.5 \mathrm{~T} \leq \mu_{0} \mathrm{H} \leq 1.5 \mathrm{~T}$ for the samples showing remanent magnetization $(\mathrm{x}=0.3,0.5$ and 0.7$)$. 

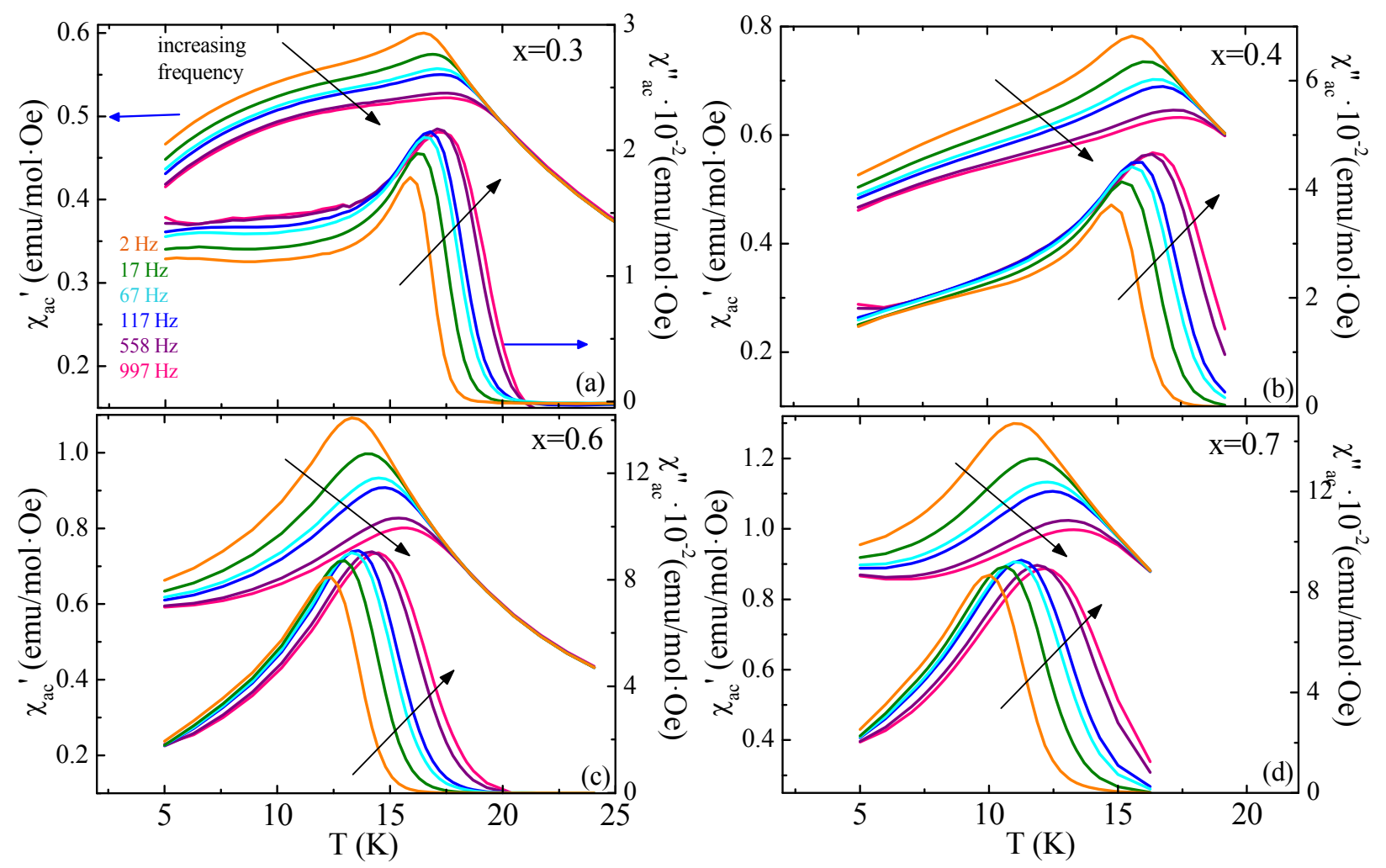

Fig. 5. ac magnetic susceptibility measurements at six different frequencies ( $v=2 \mathrm{~Hz}, 17 \mathrm{~Hz}, 67 \mathrm{~Hz}$, $117 \mathrm{~Hz}, 558 \mathrm{~Hz}$ and $997 \mathrm{~Hz})$ for $\mathrm{TbMn}_{1-\mathrm{x}} \mathrm{Sc}_{\mathrm{x}} \mathrm{O}_{3}\left(\mathrm{x}=0.3,0.4,0.6\right.$ and 0.7) with $\mathrm{h}_{0}=4$ Oe. 


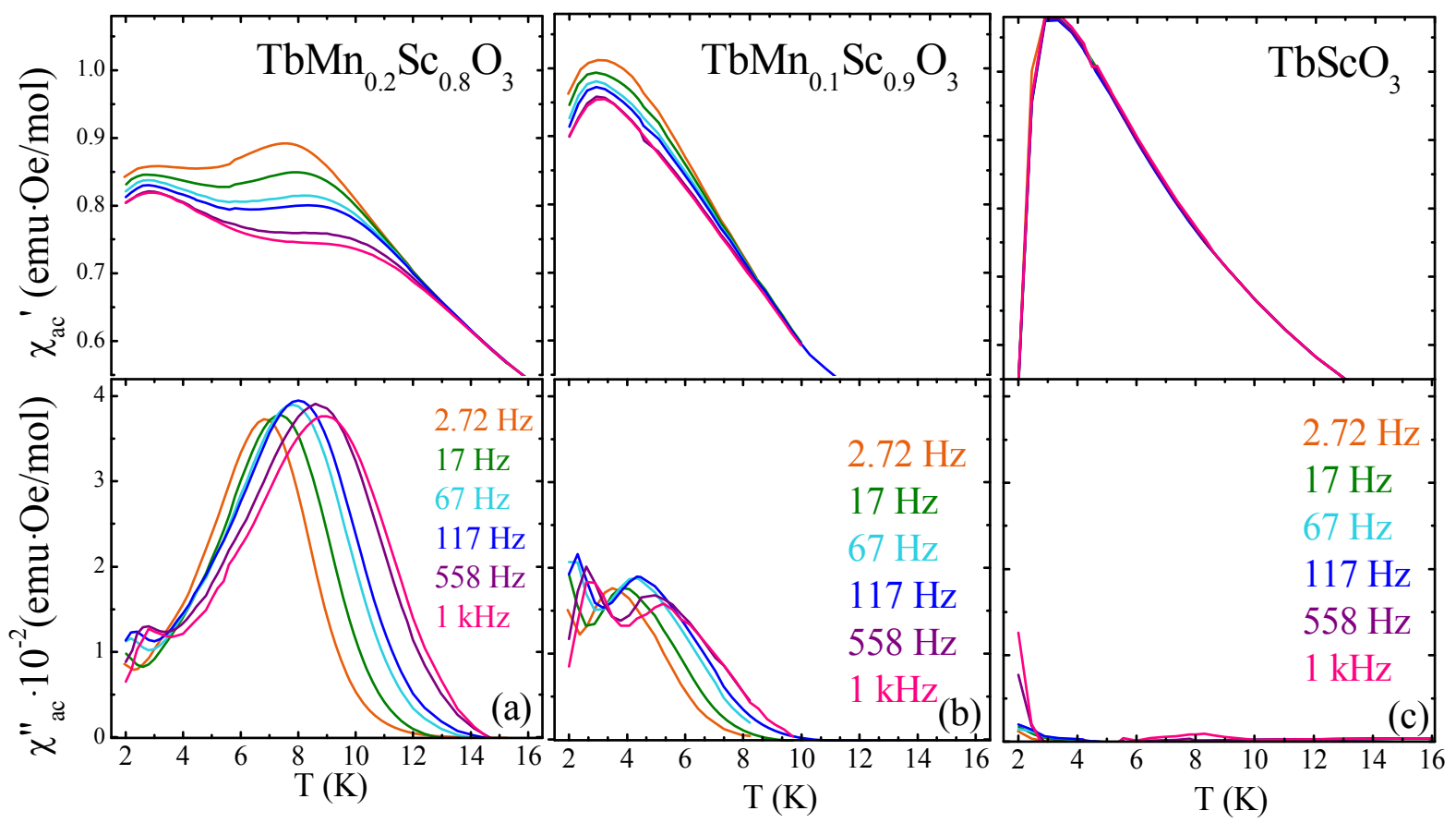

Fig. 6. Low temperature ac susceptibility measurements at six different frequencies for higher doping in $\mathrm{TbMn}_{1-\mathrm{x}} \mathrm{Sc}_{\mathrm{x}} \mathrm{O}_{3}$ compounds $(\mathrm{x}=0.8,0.9,1), \mathrm{H}_{0}=4$ Oe.

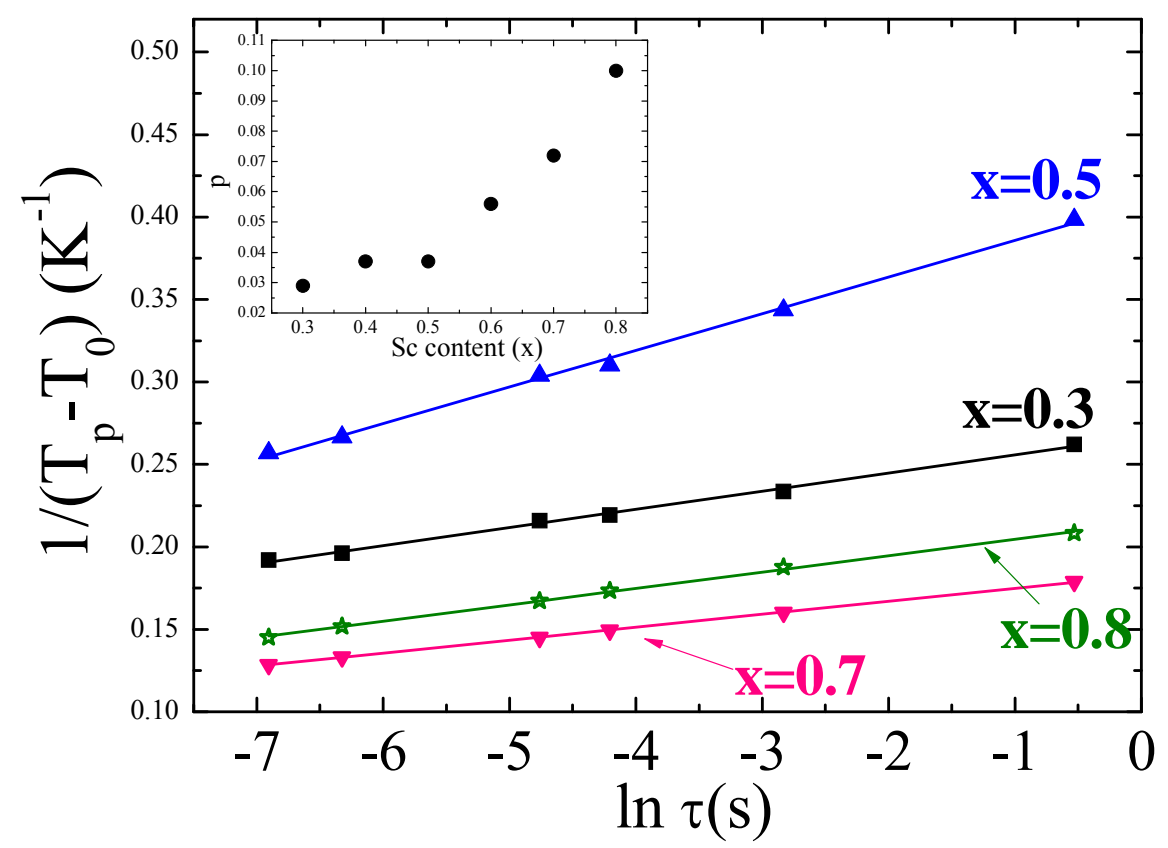

Fig 7. Frequency dependence of $\mathrm{T}_{\mathrm{p}}$ fitted to a Vogel-Fulcher law for $\mathrm{TbMn}_{1-\mathrm{x}} \mathrm{Sc}_{\mathrm{x}} \mathrm{O}_{3}(\mathrm{x}=0.3,0.5,0.7$ and 0.8) samples. Inset: values of the $p$ parameter $\left(p=\Delta T_{p} / T_{p} \cdot \Delta(\log \omega)\right)$ for the different samples of the series showing dynamic magnetic behaviour $(0.4 \leq \mathrm{x} \leq 0.8)$. 


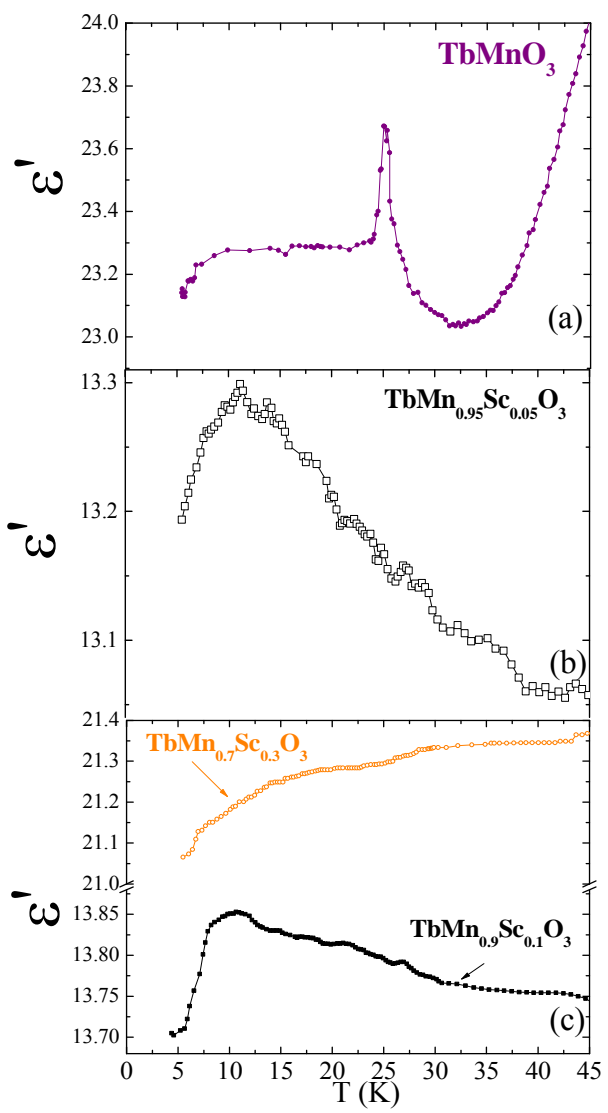

Fig. 8. Dielectric constant at $1 \mathrm{kHz}$ and low temperatures for (a) $\mathrm{TbMnO}_{3}$, (b) $\mathrm{x}=0.05$, (c) $\mathrm{x}=0.1$ and 0.3 . 


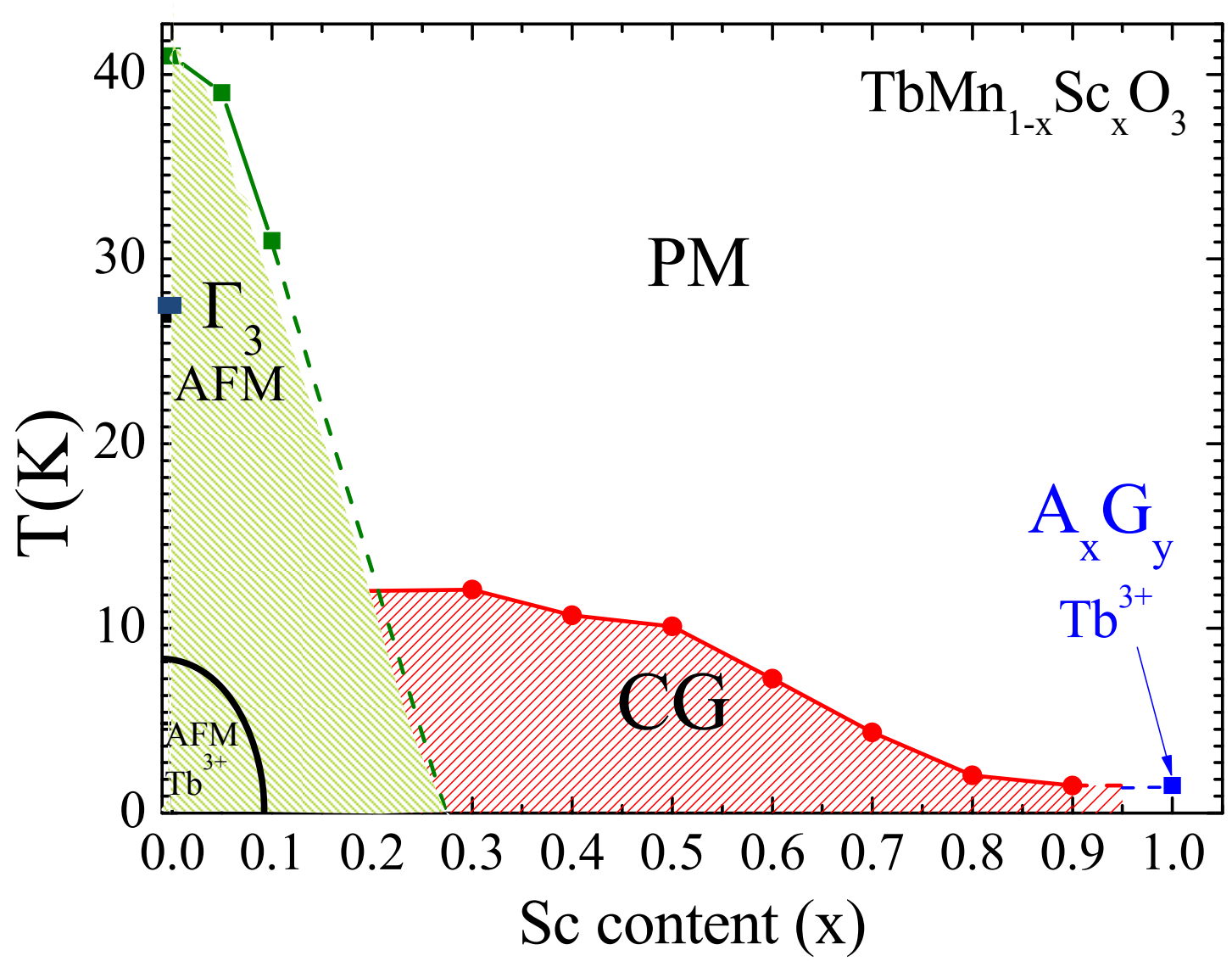

Fig. 9. Phase diagram of $\mathrm{TbMn}_{1-\mathrm{x}} \mathrm{Sc}_{\mathrm{x}} \mathrm{O}_{3}$ series, where $\mathrm{PM}$ stands for paramagnetic, AFM antiferromagnetic and CG cluster glass. The black square represents the FE and non-collinear magnetic phase transition of $\mathrm{TbMnO}_{3}$. 